\title{
Motion of Nonholonomous Rheonomous Systems in the Lagrangian Formalism
}

\author{
F. Talamucci \\ DIMAI, Dipartimento di Matematica e Informatica "Ulisse Dini”, Università Degli Studi di Firenze, Florence, Italy \\ Email: federico.talamucci@math.unifi.it
}

Received 4 March 2015; accepted 19 March 2015; published 23 March 2015

Copyright (C) 2015 by author and Scientific Research Publishing Inc.

This work is licensed under the Creative Commons Attribution International License (CC BY). http://creativecommons.org/licenses/by/4.0/

c) (i) Open Access

\section{Abstract}

The main purpose of the paper consists in illustrating a procedure for expressing the equations of motion for a general time-dependent constrained system. Constraints are both of geometrical and differential type. The use of quasi-velocities as variables of the mathematical problem opens the possibility of incorporating some remarkable and classic cases of equations of motion. Afterwards, the scheme of equations is implemented for a pair of substantial examples, which are presented in a double version, acting either as a scleronomic system and as a rheonomic system.

\section{Keywords}

\section{Nonholonomous Systems, Rheonomic Constraints, Quasi-Velocites, Appell and Boltzmann-Hamel} Equations

\section{Introduction}

Nonholonomous systems are beyond a doubt more and more considered, mainly in view of the important implementations they exhibit for mechanical models.

From the mathematical point of view, the draft of the equations for such systems commonly matches the introduction of the quasi-velocities and, starting from the Euler-Poincaré equations [1], several sets of equations have been formulated.

The time-dependent case is probably more disregarded in literature: we direct here our attention especially to rheonomic systems, admitting the holonomic and nonholonomic constraints and the applied forces to depend explicitly on time.

The nonholonomous restrictions are assumed to be linear, so that the equations of motion can be written in the linear space of the admissible displacements of the system, eliminating the Lagrangian multipliers connected to the constraints. 
If on the one hand the use of quasi-velocities formally complicates calculations, on the other hand the final form of the system allows computing the equations merely by means of a list of particular matrices, once the Lagrangian function has been written and the quasi-velocities have been chosen.

We pay attention to keep separated the various contributions to the mobility of the system; the customary stationary case can be easily recovered from the general equations we will write.

An energy balance-type equation, which will be proposed in terms of the quasi-velocities, affirms the conservation of the energy in the full stationary case and shows the contributions of the different terms in the rheonomic context.

We will conclude by presenting some applications of the developed system of equations.

Most of the formal notation used onward is explained just below. For a given a list of variables $y=\left(y_{1}, \cdots, y_{n}\right)$, the operator $\nabla_{\mathbf{y}}$ will compute the gradient $\nabla_{\mathbf{y}} f=\left(\frac{\partial f}{\partial y_{1}}, \cdots, \frac{\partial f}{\partial y_{n}}\right)$ of a scalar funcion $f$, and $J_{\mathbf{y}}$ calculates the $m \times n$ Jacobian matrix of a vector $v(\mathbf{y})=\left(v_{1}(\mathbf{y}), \cdots, v_{m}(\mathbf{y})\right):\left(J_{\mathbf{y}} \mathbf{v}\right)_{i, j}=\frac{\partial v_{i}}{\partial y_{j}}, i=1, \cdots, m, j=1, \cdots, n$.

Anywhere, vectors are in bold type and are meant as columns: row vectors will be written by means of the transposition symbol ${ }^{\mathrm{T}}$. Moreover, $\mathbf{0}_{n}$ is the null column vector $\left(\begin{array}{c}0 \\ \cdots \\ 0\end{array}\right) \in \mathbb{R}^{n}, \mathbb{O}_{n, m}$ is the $n \times m$ null matrix, $\mathbb{O}_{n}$ the $n \times m$ null matrix and $\mathbb{I}_{n}$ the unit matrix of size $n$.

\section{Modelling the System}

The theoretical frame we point and expand is contained in [2].

Let us consider a system of $n$ point particles $\left(P_{1}, m_{1}\right), \cdots,\left(P_{n}, m_{n}\right)$ restricted both by $\mu$ geometrical constraints and by $v$ kinematic constraints, $\mu \geq 0, v \geq 0, \mu+v<3 n$ :

$$
\begin{aligned}
& \mathbf{Y}(\mathbf{X}, t)=\mathbf{0}_{\mu} \\
& \mathcal{G}(\mathbf{X}, t) \dot{\mathbf{X}}+\mathbf{G}_{1}(\mathbf{X}, t)=\mathbf{0}_{v}
\end{aligned}
$$

where $\mathbf{X} \in \mathbb{R}^{3 n}$ is the representative vector of the system and, for each fixed $t, \quad \mathbf{Y}=\left(Y_{1}, \cdots, Y_{\mu}\right): \mathbb{R}^{3 n} \rightarrow \mathbb{R}^{\mu}, \mathcal{G}$ is a matrix of size $3 n \times v, \mathbf{G}_{1}$ a vector in $\mathbb{R}^{v}$. The constraint equations are assumed to be independent:

$$
\operatorname{rank} J_{\mathbf{X}} \mathbf{Y}=\mu, \quad \operatorname{rank} \mathcal{G}=v
$$

We first make use of the $v$ integer relations (1) in order to write the system configuration by means of the parametrisation $\mathbf{X}=\mathbf{X}(\mathbf{q}, t)$, where $\mathbf{q}=\left(q_{1}, \cdots, q_{\ell}\right) \in \mathcal{Q} \subseteq \mathbb{R}^{\ell}, \ell=3 n-\mu$ are the local Lagrangian coordinates. The velocity of the system $\dot{\mathbf{X}}=\left(J_{\mathbf{q}} \mathbf{X}\right) \dot{\mathbf{q}}+\frac{\partial \mathbf{X}}{\partial t}$ agrees with (1), but it must be consistent also with the differential constraints (2) which are rewritten, in terms of the Lagrangian coordinates $\mathbf{q}$ and of the generalized velocities $\dot{\mathbf{q}}$, as

$$
\alpha(\mathbf{q}, t) \dot{\mathbf{q}}+\boldsymbol{\beta}(\mathbf{q}, t)=0_{v}, \quad \alpha(\mathbf{q}, t)=\mathcal{G}(\mathbf{X}(\mathbf{q}, t), t)\left(J_{\mathbf{q}} \mathbf{X}\right) \in \mathbb{R}^{v \times \ell}, \quad \boldsymbol{\beta}(\mathbf{q}, t)=\mathcal{G} \frac{\partial \mathbf{X}}{\partial t}+\mathbf{G}_{1} \in \mathbb{R}^{v}
$$

and $\boldsymbol{\beta}=\mathbf{0}_{v}$ in case of fixed constraints. The dynamics of the system is summarized in $\mathbb{R}^{3 n}$ by $\dot{\mathbf{Q}}-\mathbf{F}-\Phi=\mathbf{0}_{3 n}$, where $\mathbf{Q}=\left(m_{s} \dot{P}_{1}, \cdots, m_{n} \dot{P}_{n}\right)$ represents the momentum of the system, $\mathbf{F}, \Phi \in \mathbb{R}^{3 n}$ respectively all active forces and all constraint reactions (the $i$-th triplet concerning $P_{i}$ ). The virtual displacements of the system at each time $t$ and at each position $\mathbf{X}$ are the vectors in $\dot{\mathbf{X}} \in \mathbb{R}^{3 n}$ such that [2]

$$
\left\{\begin{array}{l}
J_{\mathbf{X}} \mathbf{Y}(\mathbf{X}, t) \hat{\mathbf{X}}=\mathbf{0}_{\mu} \\
\mathcal{G}(\mathbf{X}, t) \hat{\mathbf{X}}=\mathbf{0}_{v}
\end{array}\right.
$$


giving in each $\mathbf{X}, t$ the $3 n-(\mu+v)$ dimensional linear space

$$
\mathcal{V}=\left(\operatorname{span}\left\langle\nabla_{\mathbf{X}} Y_{1}, \cdots, \nabla_{\mathbf{X}} Y_{\mu}, \mathcal{G}_{1}, \cdots, \mathcal{G}_{v}\right\rangle\right)^{\perp} \subset \mathbb{R}^{3 n},
$$

where $\mathcal{G}_{1}, \cdots, \mathcal{G}_{v}$ are the rows of $\mathcal{G}$. At the same time, the assumption of smooth constraints $\Phi \cdot \hat{\dot{\mathbf{X}}}=0$ $\forall \dot{\mathbf{X}} \in \mathcal{V}$ make us write

$$
\Phi=\sum_{j=1}^{\mu} \lambda_{j} \nabla_{\mathbf{x}} f_{j}+\sum_{j=1}^{v} \bar{\lambda}_{j} \mathcal{G}_{j}
$$

where $\lambda_{j}, \bar{\lambda}_{j}$ are unknown multipliers.

The projection of the dynamics equation on the subspace generated by the $\ell$ vectors $\frac{\partial \mathbf{X}}{\partial q_{i}}, i=1, \cdots, \ell$ (the columns of $J_{\mathbf{q}} \mathbf{X}$ ), although such as space strictly includes $\mathcal{V}$, if $v>0$, is anyhow noteworthy:

$$
\left(J_{\mathbf{q}} \mathbf{X}\right)^{\mathrm{T}}(\dot{\mathbf{Q}}-\mathbf{F}-\Phi)=\frac{\mathrm{d}}{\mathrm{d} t}\left(\nabla_{\dot{\mathbf{q}}} \mathcal{L}\right)-\nabla_{\mathbf{q}} \mathcal{L}-\alpha^{\mathrm{T}} \bar{\lambda}=\mathbf{0}_{\ell}, \quad \bar{\lambda}=\left(\begin{array}{l}
\bar{\lambda}_{1} \\
\cdots \\
\bar{\lambda}_{v}
\end{array}\right)
$$

where we assumed $\mathbf{F}=\nabla_{\mathbf{X}} \mathcal{U}(\mathbf{X}, t)$ and we defined the Lagrangian function

$$
\mathcal{L}=T+U, \quad T(\mathbf{q}, \dot{\mathbf{q}}, t)=\frac{1}{2} \dot{\mathbf{q}} \cdot A(\mathbf{q}, t) \dot{\mathbf{q}}+\mathbf{b}(\mathbf{q}, t) \cdot \dot{\mathbf{q}}+c(\mathbf{q}, t)
$$

with $A$ symmetric and positive definite matrix of size $\ell$ and $U(\mathbf{q}, t)=\mathcal{U}(\mathbf{x}(\mathbf{q}, t), t)$. The $\ell$ Equation (7) written for the $\ell+v$ unknown quantities $q_{1}, \cdots, q_{\ell}, \bar{\lambda}_{1}, \cdots, \bar{\lambda}_{v}$ have to be considered together with the $v$ Equations (4).

In order to improve (7), we see from (4) and (5) that $\mathcal{V}$ (virtual displacements) is the set of vectors

$$
\hat{\dot{\mathbf{X}}}=\sum_{i=1}^{\ell} \gamma_{i} \frac{\partial \hat{\dot{\mathbf{X}}}}{\partial q_{i}} \text { such that } \alpha \gamma=\mathbf{0}_{v}, \quad \gamma=\left(\begin{array}{c}
\gamma_{1} \\
\cdots \\
\gamma_{\ell}
\end{array}\right) .
$$

Owing to (3) and recalling (4), it is rank $\alpha=v$, hence the solution of the come last linear system, which explicitly writes $\sum_{j=1}^{\ell} \alpha_{i, j}(\mathbf{q}, t) \gamma_{j}=0, i=1, \cdots, v$ is

$$
\gamma_{i}=\sum_{j=1}^{\sigma} \Gamma_{i, j}(\mathbf{q}, t) \eta_{j}, \quad i=1, \cdots, \ell
$$

with $\Gamma_{i, j}$ appropriate coefficients and $\eta=\left(\eta_{1}, \cdots, \eta_{\sigma}\right)$ arbitrary factors in $\mathbb{R}^{\sigma}, \sigma=\ell-v$. We conclude that $\hat{\dot{\mathbf{X}}}=\sum_{i=1}^{\ell} \sum_{j=1}^{\sigma} \Gamma_{i, j} \eta_{j} \frac{\partial \hat{\mathbf{X}}}{\partial q_{i}}$, or, equivalently, the $\sigma$ vectors $\sum_{i=1}^{\ell} \Gamma_{i, k} \frac{\partial \mathbf{X}}{\partial q_{i}}, k=1, \cdots, \sigma$ form a basis for $\mathcal{V}$.

At this stage, calling $\Gamma$ the matrix of size $\ell \times \sigma$ and elements $\Gamma_{i, j}$ and noticing that the columns of $\left(J_{\mathbf{q}} \mathbf{X}\right) \Gamma$ give the basis for $\mathcal{V}$, the projection of the dynamics equation on $\mathcal{V}$ gives, by virtue also of (6):

$$
\left(\left(J_{\mathbf{q}} \mathbf{X}\right) \Gamma\right)^{\mathrm{T}}(\dot{\mathbf{Q}}-\mathbf{F}-\Phi)=\Gamma^{\mathrm{T}}\left(J_{\mathbf{q}} \mathbf{X}\right)^{\mathrm{T}}(\dot{\mathbf{Q}}-\mathbf{F})=\Gamma^{\mathrm{T}}\left(\frac{\mathrm{d}}{\mathrm{d} t}\left(\nabla_{\dot{\mathbf{q}}} \mathcal{L}\right)-\nabla_{\mathbf{q}} \mathcal{L}\right)=\mathbf{0}_{\sigma}
$$

where the effect of the nonholonomic constraints (through $\Gamma$ ) on the ordinary Lagrangian equations for holonomic systems is evident (in the absence of (2), say $v=0$, both (10) and (7) are $\frac{\mathrm{d}}{\mathrm{d} t}\left(\nabla_{\mathbf{q}} \mathcal{L}\right)-\nabla_{\mathbf{q}} \mathcal{L}=\mathbf{0}_{\ell}$ ).

The $\sigma$ differential Equation (10) are for the $\ell$ unknown quantities $\mathbf{q}$ and they have to be combined to- 
gether with the $v$ Equation (4). With respect to (7), they have the advantage of not exhibiting the multipliers $\bar{\lambda}$.

Remark 2.1 Either Equation (7) or (10) can be employed not necessarily for discrete systems of point particles: once the Lagrangian coordinates have been selected and the Lagrangian function has been written, they can be the same calculated.

The expedience of introducing quasi-velocities (or pseudovelocities) which have to be chosen in a suitable way in order to disentangle the mathematical problem, is by custom performed in nonholonomic systems.

Following the adopted standpoint, the definition of the quasi-velocities steps in establishing a specific (and convenient) connection between $\eta$ and $\dot{\mathbf{q}}$

$$
\eta_{1}=\sum_{j=1}^{\ell} z_{1, j}(\mathbf{q}, t) \dot{q}_{j}+\psi_{1}(\mathbf{q}, t), \cdots, \eta_{\sigma}=\sum_{j=1}^{\ell} z_{\sigma, j}(\mathbf{q}, t) \dot{q}_{j}+\psi_{\sigma}(\mathbf{q}, t) \text { or } \boldsymbol{\eta}=Z(\mathbf{q}, t) \dot{\mathbf{q}}+\psi(\mathbf{q}, t)
$$

where $z_{i, j}$ are required to guarantee that the square matrix of size $\ell\left(\begin{array}{ccc}z_{1,1} & \cdots & z_{1, \ell} \\ \cdots & \cdots & \cdots \\ z_{\sigma, 1} & \cdots & z_{\sigma, \ell} \\ \alpha_{1,1} & \cdots & \alpha_{1, \ell} \\ \cdots & \cdots & \cdots \\ \alpha_{v, 1} & \cdots & \alpha_{v, \ell}\end{array}\right)=\left(\begin{array}{l}Z \\ \alpha\end{array}\right)$ is invertible. In this way, each set of kinetic variables $\dot{\mathbf{q}}$ is linked to a singular set of quasi-velocities $\eta$, and vice versa. More precisely, (11) and (4) give

$$
\left(\begin{array}{l}
Z \\
\alpha
\end{array}\right) \dot{\mathbf{q}}=\left(\begin{array}{c}
\eta-\psi \\
-\boldsymbol{\beta}
\end{array}\right) \text { and } \quad \dot{\mathbf{q}}=\left(\begin{array}{l}
Z \\
\alpha
\end{array}\right)^{-1}\left(\begin{array}{c}
\eta-\psi \\
-\boldsymbol{\beta}
\end{array}\right)=\left(\begin{array}{ll}
\Gamma & \Theta
\end{array}\right)\left(\begin{array}{c}
\eta-\psi \\
-\boldsymbol{\beta}
\end{array}\right)
$$

where $\Gamma$ is the same as (9) and $\Theta(\mathbf{q}, t)$ is a $\ell \times v$ matrix. The first system in (12) shows both the selection on the coordinates $\dot{\mathbf{q}}$ of the tangent space $J_{\mathbf{q}} \mathbf{X}$ necessary to fulfill the restrictions on the system's velocity (leading to the subspace $\mathcal{V}$ ) and the kinematic conditions themselves. (12)

In order to express (10) as a function of the variables $\mathbf{q}, \eta$ and to eliminate $\dot{\mathbf{q}}$, it suffices to extract from

$$
\dot{\mathbf{q}}(\mathbf{q}, \eta, t)=\Gamma(\mathbf{q}, t)(\eta-\psi(\mathbf{q}, t))-\Theta(\mathbf{q}, t) \boldsymbol{\beta}(\mathbf{q}, t)
$$

and to define

$$
\begin{aligned}
\tilde{\mathcal{L}}(\mathbf{q}, \eta, t) & =\mathcal{L}(\mathbf{q}, \dot{\mathbf{q}}(\mathbf{q}, \eta, t), t) \\
& =\frac{1}{2}\left[(\eta-\psi) \cdot A_{\Gamma}(\eta-\psi)+\boldsymbol{\beta} \cdot A_{\Theta} \boldsymbol{\beta}-2(\eta-\psi) \cdot A_{\Gamma, \Theta} \boldsymbol{\beta}\right]+\mathbf{b} \cdot(\Gamma(\eta-\psi)-\Theta \boldsymbol{\beta})+c+U,
\end{aligned}
$$

where

$$
A_{\Gamma}(\mathbf{q}, t)=\Gamma^{\mathrm{T}} A \Gamma, \quad A_{\Gamma, \Theta}(\mathbf{q}, t)=\Gamma^{\mathrm{T}} A \Theta, \quad A_{\Theta}(\mathbf{q}, t)=\Theta^{\mathrm{T}} A \Theta
$$

By using the formulae (see (11))

$$
\nabla_{\mathbf{q}} \mathcal{L}=Z^{\mathrm{T}} \nabla_{\eta} \tilde{\mathcal{L}}, \quad \nabla_{\mathbf{q}} L=\nabla_{\mathbf{q}} \tilde{\mathcal{L}}+\left.\left(J_{\mathbf{q}}^{\mathrm{T}} \eta\right)\right|_{\dot{\mathbf{q}}=\Gamma(\eta-\psi)-\Theta \beta} \nabla_{\eta} \tilde{\mathcal{L}}
$$

where $J_{\mathbf{q}}^{\mathrm{T}} \eta(\mathbf{q}, \eta, t)$ is the $\ell \times \sigma$ matrix whose elements are, for each $i=1, \cdots, \ell, j=1, \cdots, \sigma$

$$
\left(\left.\left(J_{\mathbf{q}}^{\mathrm{T}} \eta\right)\right|_{\mathbf{q}=\Gamma(\eta-\psi)-\Theta \boldsymbol{\beta}}\right)_{i, j}=\sum_{k=1}^{\ell} \frac{\partial \mathbf{z}_{j, k}}{\partial \boldsymbol{q}_{i}}\left(\sum_{s=1}^{\sigma} \Gamma_{k, s}\left(\eta_{s}-\psi_{s}\right)-\sum_{p=1}^{v} \Theta_{k, p} \boldsymbol{\beta}_{p}\right)+\frac{\partial \psi_{j}}{\partial q_{i}}
$$

we can write (10) in terms of the demanded variables (we use $Z \Gamma=I_{\sigma}$, see (12)): 


$$
\frac{\mathrm{d}}{\mathrm{d} t}\left(\nabla_{\eta} \tilde{\mathcal{L}}\right)-\Gamma^{\mathrm{T}} \nabla_{\mathbf{q}} \tilde{\mathcal{L}}+\Gamma^{\mathrm{T}}\left(\dot{Z}^{\mathrm{T}}-J_{\mathbf{q}}^{\mathrm{T}} \eta\right) \nabla_{\eta} \tilde{\mathcal{L}}=\mathbf{0}_{\sigma}
$$

Remark 2.2 Multiplying both sides of (17) by $\eta$ and performing the customary steps leading to the energy balance one finds

$$
\begin{aligned}
& \frac{\mathrm{d}}{\mathrm{d} t}\left(\eta \cdot \nabla_{\eta} \tilde{\mathcal{L}}-\tilde{\mathcal{L}}\right)+\frac{\partial \tilde{\mathcal{L}}}{\partial t}-(\Theta \boldsymbol{\beta}+\Gamma \psi) \cdot\left[\nabla_{\mathbf{q}} \tilde{\mathcal{L}}+\left(J_{\mathbf{q}} \eta+\frac{\partial Z}{\partial t}\right)^{\mathrm{T}} \nabla_{\eta} \tilde{\mathcal{L}}\right] \\
& +\left\{\frac{\partial Z}{\partial t} \Gamma \eta+\frac{\partial \psi}{\partial t}-Z \frac{\mathrm{d}}{\mathrm{d} t}(\Theta \boldsymbol{\beta}+\Gamma \psi)\right\} \cdot \nabla_{\eta} \tilde{\mathcal{L}}=0
\end{aligned}
$$

In the stationary circumstance $\boldsymbol{\beta}=\mathbf{0}, \boldsymbol{\psi}=\mathbf{0}, \tilde{\mathcal{L}}=\tilde{\mathcal{L}}(\mathbf{q}, \boldsymbol{\eta})$ and $Z=Z(\mathbf{q})$ the Legendre transform $\eta \cdot \nabla_{\eta} \tilde{\mathcal{L}}-\tilde{\mathcal{L}}$ of $\tilde{\mathcal{L}}$ is conserved.

Our next step is writing (17) explicitly, sorting the terms in a suitable way: we start from the calculation

$$
\begin{aligned}
\nabla_{\eta} \tilde{\mathcal{L}}(\mathbf{q}, \boldsymbol{\eta}, t)= & A_{\Gamma}(\boldsymbol{\eta}-\boldsymbol{\psi})-A_{\Gamma, \Theta} \boldsymbol{\beta}+\Gamma^{\mathrm{T}} \mathbf{b}, \\
\nabla_{\mathbf{q}} \tilde{\mathcal{L}}(\mathbf{q}, \boldsymbol{\eta}, t)= & \left\{-\frac{1}{2}\left(J_{\mathbf{q}}^{\mathrm{T}} \boldsymbol{\psi}\right) A_{\Gamma}+\frac{1}{2} J_{\mathbf{q}}^{\mathrm{T}}\left(A_{\Gamma}(\boldsymbol{\eta}-\boldsymbol{\psi})\right)-J_{\mathbf{q}}^{\mathrm{T}}\left(A_{\Gamma, \Theta} \boldsymbol{\beta}\right)\right\}(\boldsymbol{\eta}-\boldsymbol{\psi}) \\
& +\left\{\frac{1}{2}\left(J_{\mathbf{q}}^{\mathrm{T}} \boldsymbol{\beta}\right) A_{\Theta}+\frac{1}{2} J_{\mathbf{q}}^{\mathrm{T}}\left(A_{\Theta} \boldsymbol{\beta}\right)+\left(J_{\mathbf{q}}^{\mathrm{T}} \boldsymbol{\psi}\right) A_{\Gamma, \Theta}\right\} \boldsymbol{\beta} \\
& +\left(J_{\mathbf{q}}^{\mathrm{T}} \mathbf{b}\right)(\Gamma(\boldsymbol{\eta}-\boldsymbol{\psi})-\Theta \boldsymbol{\beta})+\left[J_{\mathbf{q}}^{\mathrm{T}}(\Gamma(\boldsymbol{\eta}-\boldsymbol{\psi})-\Theta \boldsymbol{\beta})\right] \mathbf{b}+\nabla_{\mathbf{q}} c+\nabla_{\mathbf{q}} U .
\end{aligned}
$$

so that (17) takes the structure

$$
A_{\Gamma}(\mathbf{q}, t) \dot{\boldsymbol{\eta}}+Q(\mathbf{q}, \boldsymbol{\eta}, t)+\Lambda(\mathbf{q}, \boldsymbol{\eta}, t ; \boldsymbol{\beta}, \boldsymbol{\psi}, \mathbf{b})+N(\mathbf{q}, t ; \boldsymbol{\beta}, \boldsymbol{\psi}, \mathbf{b})-A_{\Gamma} \frac{\partial \boldsymbol{\psi}}{\partial t}-A_{\Gamma, \Theta} \frac{\partial \boldsymbol{\beta}}{\partial t}-\Gamma^{\mathrm{T}}\left(\nabla_{\mathbf{q}} c+\nabla_{\mathbf{q}} U-\frac{\partial \mathbf{b}}{\partial t}\right)=\mathbf{0}_{\sigma}
$$

Provided that $M^{(k)}$ means the $k$-th column of any matrix $M$ and defining for any $k=1, \cdots, \ell$ the operation

$$
\mathcal{D}_{k}(M)=\left[M\left(\frac{\partial Z}{\partial q_{k}}-J_{\mathbf{q}} Z^{(k)}\right) \Gamma+\frac{\partial M}{\partial q_{k}}\right]^{\mathrm{T}}
$$

for a matrix $M(\mathbf{q})$ of size $N \times \sigma$, the terms in (20) are defined by the following expressions, where $(\mathbf{v})_{k}$ means the $k$-th component of any vector $\mathbf{v}$ and $A_{\Theta, \Gamma}=A_{\Gamma, \Theta}$ :

$$
\begin{aligned}
& Q(\mathbf{q}, \boldsymbol{\eta}, t)=\left\{\sum_{k=1}^{\ell}(\Gamma \boldsymbol{\eta})_{k} \mathcal{D}_{k}\left(A_{\Gamma}\right)-\frac{1}{2} \sum_{r=1}^{\sigma} \eta_{r}\left(\left(J_{\mathbf{q}} A_{\Gamma}^{(r)}\right) \Gamma\right)^{\mathrm{T}}\right\} \boldsymbol{\eta} \\
\Lambda(\mathbf{q}, \boldsymbol{\eta}, t ; \boldsymbol{\beta}, \boldsymbol{\psi}, \mathbf{b})= & -\left\{\sum_{k=1}^{\ell}(\Gamma \boldsymbol{\psi})_{k} \mathcal{D}_{k}\left(A_{\Gamma}\right)-\frac{1}{2} \sum_{r=1}^{\sigma} \psi_{r}\left(\left(J_{\mathbf{q}} A_{\Gamma}^{(r)}\right) \Gamma\right)^{\mathrm{T}}+A_{\Gamma}\left(J_{\mathbf{q}} \boldsymbol{\psi}\right) \Gamma\right\} \eta \\
& -\left\{A_{\Gamma, \Theta}\left(J_{\mathbf{q}} \boldsymbol{\beta}\right) \Gamma-\left(A_{\Gamma, \Theta}\left(J_{\mathbf{q}} \boldsymbol{\beta}\right) \Gamma\right)^{\mathrm{T}}-\sum_{p=1}^{v} \beta_{p}\left(\left(J_{\mathbf{q}} A_{\Gamma, \Theta}^{(p)}\right) \Gamma\right)^{\mathrm{T}}+\sum_{k=1}^{\ell}(\Theta \boldsymbol{\beta})_{k} \mathcal{D}_{k}\left(A_{\Gamma}\right)\right\} \eta \\
& -\left\{\sum_{k=1}^{\ell}(\Gamma \boldsymbol{\eta})_{k} \mathcal{D}_{k}\left(A_{\Gamma}\right)-\frac{1}{2} \sum_{r=1}^{\sigma} \eta_{r}\left(\left(J_{\mathbf{q}} A_{\Gamma}^{(r)}\right) \Gamma\right)^{\mathrm{T}}\right\} \boldsymbol{\psi}-\sum_{k=1}^{\ell}(\Gamma \boldsymbol{\eta})_{k} \mathcal{D}_{k}\left(A_{\Theta, \Gamma}\right) \boldsymbol{\beta} \\
& +\Gamma^{\mathrm{T}}\left[\left(J_{\mathbf{q}} \mathbf{b}\right)-\left(J_{\mathbf{q}} \mathbf{b}\right)^{\mathrm{T}}\right] \Gamma \eta+\left\{\sum_{k=1}^{\ell}(\Gamma \boldsymbol{\eta})_{k} \mathcal{D}_{k}(\Gamma)-\sum_{r=1}^{\sigma} \eta_{r}\left[\left(J_{\mathbf{q}} \Gamma^{(r)}\right) \Gamma\right]^{\mathrm{T}}\right\} \mathbf{b} \\
& +\left\{\frac{\partial A_{\Gamma}}{\partial t}+\left(A_{\Gamma} \frac{\partial Z}{\partial t} \Gamma\right)^{\mathrm{T}}\right\} \eta,
\end{aligned}
$$




$$
\begin{aligned}
N(\mathbf{q}, t ; \boldsymbol{\beta}, \boldsymbol{\psi}, \mathbf{b})= & \left\{A_{\Gamma, \Theta}\left(J_{\mathbf{q}} \boldsymbol{\beta}\right) \Theta-\left(A_{\Theta}\left(J_{\mathbf{q}} \boldsymbol{\beta}\right) \Gamma\right)^{\mathrm{T}}-\frac{1}{2} \sum_{p=1}^{v} \beta_{p}\left(\left(J_{\mathbf{q}} A_{\Theta}^{(p)}\right) \Gamma\right)^{\mathrm{T}}+\sum_{k=1}^{\ell}(\Theta \boldsymbol{\beta})_{k} \mathcal{D}_{k}\left(A_{\Theta, \Gamma}\right)\right\} \boldsymbol{\beta} \\
& +\left\{A_{\Gamma}\left(J_{\mathbf{q}} \boldsymbol{\psi}\right) \Gamma-\left(A_{\Gamma}\left(J_{\mathbf{q}} \boldsymbol{\psi}\right) \Gamma\right)^{\mathrm{T}}-\frac{1}{2} \sum_{r=1}^{\sigma} \psi_{r}\left(\left(J_{\mathbf{q}} A_{\Gamma}^{(r)}\right) \Gamma\right)^{\mathrm{T}}+\sum_{k=1}^{\ell}(\Gamma \boldsymbol{\psi})_{k} \mathcal{D}_{k}\left(A_{\Gamma}\right)\right\} \boldsymbol{\psi} \\
& +\left\{A_{\Gamma, \Theta}\left(J_{\mathbf{q}} \boldsymbol{\beta}\right) \Gamma-\left(A_{\Gamma, \Theta}\left(J_{\mathbf{q}} \boldsymbol{\beta}\right) \Gamma\right)^{\mathrm{T}}-\sum_{p=1}^{v} \beta_{p}\left(\left(J_{\mathbf{q}} A_{\Gamma, \Theta}^{(p)}\right) \Gamma\right)^{\mathrm{T}}+\sum_{k=1}^{\ell}(\Theta \boldsymbol{\beta})_{k} \mathcal{D}_{k}\left(A_{\Gamma}\right)\right\} \boldsymbol{\psi} \\
& +\left\{A_{\Gamma}\left(J_{\mathbf{q}} \boldsymbol{\psi}\right) \Theta-\left(A_{\Theta, \Gamma}\left(J_{\mathbf{q}} \boldsymbol{\psi}\right) \Gamma\right)^{\mathrm{T}}+\sum_{k=1}^{\ell}(\Gamma \boldsymbol{\psi})_{k} \mathcal{D}_{k}\left(A_{\Theta, \Gamma}\right)\right\} \boldsymbol{\beta} \\
& -\Gamma^{\mathrm{T}}\left[J_{\mathbf{q}} \mathbf{b}-\left(J_{\mathbf{q}} \mathbf{b}\right)^{\mathrm{T}}\right](\Gamma \boldsymbol{\psi}+\Theta \boldsymbol{\beta})+\left\{\left(\Gamma\left(J_{\mathbf{q}} \boldsymbol{\psi}\right) \Gamma\right)^{\mathrm{T}}+\left(\Theta\left(J_{\mathbf{q}} \boldsymbol{\beta}\right) \Gamma\right)^{\mathrm{T}}\right\} \mathbf{b} \\
& +\left\{\sum_{r=1}^{\sigma} \psi_{r}\left[\left(J_{\mathbf{q}} \Gamma^{(r)}\right) \Gamma\right]^{\mathrm{T}}+\sum_{p=1}^{v} \beta_{v}\left[\left(J_{\mathbf{q}} \Theta^{(p)}\right) \Gamma\right]^{\mathrm{T}}-\sum_{k=1}^{\ell}(\Gamma \boldsymbol{\psi}+\Theta \boldsymbol{\beta})_{k} \mathcal{D}_{k}(\Gamma)\right\} \mathbf{b} \\
& -\left\{\frac{\partial A_{\Gamma, \Theta}}{\partial t}+\left(A_{\Theta, \Gamma} \frac{\partial Z}{\partial t} \Gamma\right)^{\mathrm{T}}\right\} \boldsymbol{\beta}-\left\{\frac{\partial A_{\Gamma}}{\partial t}+\left(A_{\Gamma} \frac{\partial Z}{\partial t} \Gamma\right)^{\mathrm{T}}\right\} \boldsymbol{\psi}+\left\{\frac{\partial \Gamma^{\mathrm{T}}}{\partial t}+\left(\Gamma \frac{\partial Z}{\partial t} \Gamma\right)^{\mathrm{T}}\right\} \mathbf{b .}
\end{aligned}
$$

Equation (20) is sorted on the strength of the quasi-velocities $\boldsymbol{\eta}: Q$ is quadratic with respect to $\eta_{1}, \cdots, \eta_{\sigma}$, $\Lambda$ is linear with respect to the same variables and $Z$ does not contain $\eta$.

Since $A$ is a positive-definite square matrix and $\operatorname{rank} \Gamma=\sigma$, even $A_{\Gamma}=\Gamma^{\mathrm{T}} A \Gamma$ is a positive-definite $\sigma \times \sigma$ symmetric matrix. Hence, system (20) + (13) can be written in the normal form $\left(\begin{array}{l}\dot{\boldsymbol{\eta}} \\ \mathbf{q}\end{array}\right)=\mathbf{Y}(\boldsymbol{\eta}, \mathbf{q}, t)$, where $\mathbf{Y}$ is a list of $\ell+\sigma$ functions, whose regularity allows us to apply the standard theorems on existence and uniqueness of solutions to first-order equations with given initial conditions.

Before commenting Equation (20), we remark that the $\sigma \times N$ entries of the matrix $\mathcal{D}_{k}(M)$ defined in (21) are, for each $k=1, \cdots, \ell$ :

$$
\left(\mathcal{D}_{k}(M)\right)_{i, j}=\sum_{h=1}^{\ell} \sum_{r=1}^{\sigma} M_{j, r}\left(\frac{\partial z_{r, h}}{\partial q_{k}}-\frac{\partial z_{r, k}}{\partial q_{h}}\right) \Gamma_{h, i}+\frac{\partial M_{j, i}}{\partial q_{k}}, \quad i=1, \cdots, \sigma, j=1, \cdots, N
$$

We see now that a certain number of significant cases are encompassed by (20):

- merely geometric constraints, corresponding to $\sigma=\ell, v=0$, so that (4) are not present and all the terms containing $\boldsymbol{\beta}, \Theta$ and the related quantities $A_{\Theta} \quad A_{\Gamma, \Theta}$ must be dropped in (20). Furthermore:

$\circ$ selecting $\boldsymbol{\eta}=\dot{\mathbf{q}}$ (quasi-velocities are the generalized velocities) in (11) and (13) means

$$
Z=\Gamma=\mathbb{I}_{\ell}, \quad A_{\Gamma}=A, \quad \boldsymbol{\psi}=\mathbf{0}_{\ell}
$$

so that in (20) are written with as

$$
Q=\sum_{k=1}^{\ell}\left\{\dot{q}_{k} \mathcal{D}_{k}(A)-\frac{1}{2} \dot{q}_{k}\left(J_{\mathbf{q}} A^{(k)}\right)^{\mathrm{T}}\right\} \dot{\mathbf{q}}, \quad \Lambda=\left\{J_{q} \mathbf{b}-\left(J_{q} \mathbf{b}\right)^{\mathrm{T}}+\frac{\partial A}{\partial t}\right\} \dot{\mathbf{q}}, \quad N=\mathbf{0}
$$

thus the Lagrangian equations for geometric constraints (bearing in mind (22))

$$
\sum_{r=1}^{\ell} a_{i, r} \ddot{q}_{r}+\sum_{r, s=1}^{\ell}\left(\frac{\partial a_{i, r}}{\partial q_{s}}-\frac{1}{2} \frac{\partial a_{r, s}}{\partial q_{i}}\right) \dot{q}_{r} \dot{q}_{s}+\sum_{r=1}^{\ell}\left(\frac{\partial b_{i}}{\partial q_{r}}-\frac{\partial b_{r}}{\partial q_{i}}+\frac{\partial a_{i, r}}{\partial t}\right) \dot{q}_{r}-\frac{\partial c}{\partial q_{i}}-\frac{\partial U}{\partial q_{i}}+\frac{\partial b_{i}}{\partial t}=0
$$

$i=1, \cdots, \ell$, are achieved.

$\circ$ establishing (11) as $\boldsymbol{\eta}=\nabla_{\dot{\mathbf{q}}} \mathcal{L}=A \dot{\mathbf{q}}+\mathbf{b}$ (quasi-velocities are the generalized momenta) means

$$
Z=A, \quad \Gamma=A^{-1}, \quad A_{\Gamma}=\Gamma, \quad \psi=\mathbf{b}
$$

In this case (13) together with (20) are the Hamiltonian equations for 


$$
H(\mathbf{q}, \boldsymbol{\eta}, t)=\boldsymbol{\eta} \cdot \dot{\mathbf{q}}(\mathbf{q}, \boldsymbol{\eta}, t)-\tilde{\mathcal{L}}(\mathbf{q}, \boldsymbol{\eta}, t)=\frac{1}{2} \Gamma(\boldsymbol{\eta}-\mathbf{b}) \cdot(\boldsymbol{\eta}-\mathbf{b})-c-U:
$$

indeed the first one is $\dot{\mathbf{q}}=\Gamma(\boldsymbol{\eta}-\mathbf{b})=\nabla_{\eta} H$, whereas (20) reduces to

$$
\Gamma(\mathbf{q}, t) \dot{\boldsymbol{\eta}}+Q(\mathbf{q}, \boldsymbol{\eta}, t)+\Lambda(\mathbf{q}, \boldsymbol{\eta}, t ; \mathbf{b})+N(\mathbf{q}, t ; \mathbf{b})-\Gamma\left(\nabla_{\mathbf{q}} c+\nabla_{\mathbf{q}} U\right)=\mathbf{0}_{\ell}
$$

with

$$
\begin{gathered}
Q(\mathbf{q}, \boldsymbol{\eta}, t)=-\sum_{k=1}^{\ell}(\Gamma \boldsymbol{\eta})_{k} \Gamma\left(J_{\mathbf{q}} A^{(k)}\right)^{\mathrm{T}} \Gamma \boldsymbol{\eta}-\frac{1}{2} \sum_{k=1}^{\ell} \eta_{k} \Gamma\left(J_{\mathbf{q}} \Gamma^{(k)}\right)^{\mathrm{T}} \boldsymbol{\eta} \\
\Lambda(\mathbf{q}, \boldsymbol{\eta}, t ; \boldsymbol{\beta}, \psi, \mathbf{b})=-\Gamma\left(J_{\mathbf{q}} \mathbf{b}\right)^{\mathrm{T}} \Gamma \boldsymbol{\eta}-\frac{1}{2} \sum_{k=1}^{\ell} \eta_{k} \Gamma\left(J_{\mathbf{q}} \Gamma^{(k)}\right)^{\mathrm{T}} \mathbf{b}+\sum_{k=1}^{\ell}(\Gamma \mathbf{b})_{k} \Gamma\left(J_{\mathbf{q}} A^{(k)}\right)^{\mathrm{T}} \Gamma \boldsymbol{\eta} \\
N(\mathbf{q}, t ; \mathbf{b})=\Gamma\left(J_{\mathbf{q}} \mathbf{b}\right)^{\mathrm{T}} \Gamma \mathbf{b}+\frac{1}{2} \sum_{k=1}^{\ell} b_{k} \Gamma\left(J_{\mathbf{q}} \Gamma^{(k)}\right)^{\mathrm{T}} \Gamma
\end{gathered}
$$

(actually from $\Gamma A=\mathbb{I}_{\ell}$ one deduces $\mathcal{D}_{k}(\Gamma)=-\Gamma\left(J_{\mathbf{q}} A^{(k)}\right)^{\mathrm{T}} \Gamma$ and $\Gamma \frac{\partial A}{\partial t}=-\frac{\partial \Gamma}{\partial t} A$ so that, also considering $\mathbf{b}=\boldsymbol{\psi}$, many terms are cancelled).

Since $\left(J_{\mathbf{q}} A^{(k)}\right)^{\mathrm{T}} \Gamma^{(h)}=-\left(J_{\mathbf{q}} \Gamma^{(h)}\right)^{\mathrm{T}} A^{(k)}$ for any $h, k=1, \cdots, \ell$, it is

$$
Q+\Lambda+N=\Gamma\left\{\frac{1}{2} \sum_{k=1}^{\ell}\left(\eta_{k}-b_{k}\right)\left(J_{\mathbf{q}} \Gamma^{(k)}\right)^{\mathrm{T}}-\left(J_{\mathbf{q}} \mathbf{b}\right)^{\mathrm{T}} \Gamma\right\}(\boldsymbol{\eta}-\mathbf{b})=\Gamma \nabla_{q}\left(\frac{1}{2} \Gamma(\boldsymbol{\eta}-\mathbf{b}) \cdot(\boldsymbol{\eta}-\mathbf{b})\right)
$$

therefore (23) is $\Gamma \dot{\boldsymbol{\eta}}=-\Gamma \nabla_{\mathbf{q}} H$, as stated.

- Stationary case, where the different contributions producing the dependence on $t$ must be dropped. If one is dealing with a scleronomic system (covering many of common instances), the constraints (1), (2) reduce to

$$
\begin{gathered}
\mathbf{Y}(\mathbf{X})=\mathbf{0}_{\mu} \\
\mathcal{G}(\mathbf{X}) \mathbf{X}=\mathbf{0}_{v}
\end{gathered}
$$

Conditions (24) entail $\mathbf{X}=\mathbf{X}(\mathbf{q})$ and $\mathcal{L}(\mathbf{q}, \dot{\mathbf{q}})=\frac{1}{2} \dot{\mathbf{q}} \cdot A(\mathbf{q}) \dot{\mathbf{q}}+U(\mathbf{q})$ (if even the forces are independent of time), on the other hand (25) implies $\boldsymbol{\beta}=\mathbf{0}$.

Equation (11), if one reasonably chooses $\psi=\mathbf{0}$ and $Z$ independent of $t$ (otherwise, changes will be obvious), is $\boldsymbol{\eta}=Z(\mathbf{q}) \dot{\mathbf{q}}$. Since $\Lambda=N=\mathbf{0}_{\sigma}$, system (20) + (13) drastically simplifies to

$$
\left\{\begin{array}{l}
A_{\Gamma}(\mathbf{q}) \dot{\boldsymbol{\eta}}+Q(\mathbf{q}, \boldsymbol{\eta})-\Gamma^{\mathrm{T}} \nabla_{\mathbf{q}} U=\mathbf{0}_{\sigma}, \\
\dot{\mathbf{q}}=\Gamma(\mathbf{q}) \boldsymbol{\eta},
\end{array}\right.
$$

or, index by index, calling $b_{i, j}$ the entries of the matrix $A_{\Gamma}, i, j=1, \cdots, \sigma$ and having in mind (22)

$$
\begin{array}{lr}
\sum_{r=1}^{\sigma} b_{i, r} \dot{\eta}_{r}+\sum_{r, s=1}^{\sigma} \mathcal{Q}_{r, s}^{(i)} \eta_{r} \eta_{s}-\sum_{h=1}^{\ell} \Gamma_{h, i} \frac{\partial U}{\partial q_{h}}=0, & i=1, \cdots, \sigma ; \\
\dot{q}_{p}=\sum_{j=1}^{\sigma} \Gamma_{p, j}\left(q_{1}, \cdots, q_{\ell}\right) \eta_{j}, & p=1, \cdots, \ell .
\end{array}
$$

where $\mathcal{Q}^{(i)}$ is, for each index $i$, the square matrix of order $\sigma$

$$
\mathcal{Q}_{r, s}^{(i)}\left(q_{1}, \cdots, q_{\ell}\right)=\sum_{h, k=1}^{\ell}\left(\Gamma_{k, i} \Gamma_{h, s} \sum_{j=1}^{\sigma} b_{r, j}\left(\frac{\partial z_{j, k}}{\partial q_{h}}-\frac{\partial z_{j, h}}{\partial q_{k}}\right)+\Gamma_{h, s} \frac{\partial b_{r, i}}{\partial q_{h}}-\frac{1}{2} \Gamma_{h, i} \frac{\partial b_{r, s}}{\partial q_{h}}\right)
$$


Equations (27) are identified with the Boltzmann-Hamel Equations (17) for the Lagrangian function $\tilde{\mathcal{L}}(\mathbf{q}, \boldsymbol{\eta})=\frac{1}{2} \boldsymbol{\eta} \cdot A_{\Gamma} \boldsymbol{\eta}+U$ (see [3] [4]). In this case the Legendre transform $\frac{1}{2} \boldsymbol{\eta} \cdot A_{\Gamma} \boldsymbol{\eta}-U$ is a first integral of motion, see Remark 1.2.

- Reduced Lagrangian function for geometric constraints: in case of $v$ cyclic variables $q_{\sigma+1}, \cdots, q_{\ell}, \sigma=\ell-v$, (4) can play the role of the $v$ relations derived from the first integral of motion $p_{i}=\frac{\partial \mathcal{L}}{\partial \dot{q}_{i}}, i=\sigma+1, \cdots, \ell$, that is $\sum_{j=1}^{\ell} a_{i, j} \dot{q}_{j}+b_{i}-p_{i}=0, \quad i=\sigma+1, \cdots, \ell$. Assuming that $\operatorname{det}\left(a_{r, s}\right) \neq 0, \quad r, s=\sigma+1, \cdots, \ell$, it is possible to acquire, according to (13), $\dot{q}_{i}=\sum_{j=1}^{\sigma} \Gamma_{i, j} \dot{q}_{j}-\Theta_{i, j}\left(b_{j}-p_{j}\right), \quad i=\sigma+1, \cdots, \ell$, where $\Gamma_{i, j}, \Theta_{i, j}$ and $b_{j}$ depend only on $\left(q_{1}, \cdots, q_{\sigma}, t\right)$. At this point, setting $\eta_{1}=\dot{q}_{1}, \cdots, \eta_{\sigma}=\dot{q}_{\sigma}$ we have, with respect to (11) and (12), $Z=\left(\begin{array}{ll}\mathbb{O}_{\sigma \times v} & \mathbb{I}_{\sigma}\end{array}\right)$ and $\Gamma_{r, s}=\delta_{r, s}$ (Kronecker's delta), $r, s=1, \cdots, \sigma$. Equation (20), which writes simply $\frac{\mathrm{d}}{\mathrm{d} t}\left(\nabla_{\eta} \tilde{\mathcal{L}}\right)=\Gamma^{\mathrm{T}} \nabla_{\mathbf{q}} \tilde{\mathcal{L}}$, are the equations of motion for the reduced Lagrangian

$$
\tilde{\mathcal{L}}\left(\mathbf{q}^{(\sigma)}, \boldsymbol{\eta}, t\right)=\mathcal{L}\left(\mathbf{q}^{(\sigma)}, \boldsymbol{\eta}, \dot{q}_{\sigma+1}\left(\mathbf{q}^{(\sigma)}, \boldsymbol{\eta}, t\right), \cdots, \dot{q}_{\ell}\left(\mathbf{q}^{(\sigma)}, \boldsymbol{\eta}, t\right), t\right),
$$

with $\boldsymbol{\eta}=\left(\eta_{1}, \cdots, \eta_{\sigma}\right)$, $\mathbf{q}^{(\sigma)}=\left(q_{1}, \cdots, q_{\sigma}\right)$; on the other hand, $\dot{q}_{i}\left(\mathbf{q}^{(\sigma)}, \eta, t\right)=\sum_{j=1}^{\sigma} \Gamma_{i, j} \eta_{j}-\Theta_{i, j}\left(b_{j}-p_{j}\right)$ for $i=\sigma+1, \cdots, \ell$, are the so called reconstruction equations.

\section{Some Applications}

We adopt now Equation (20) in order to formulate a couple of remarkable mechanical systems, each of them in a double form, as scleronomous and rheonomous model.

\subsection{Pendulum on a Skate}

Consider a system of four points $\left\{P_{F}, P_{B}, P_{S}, P_{D}\right\}, P_{F}$ and $P_{B}$ equidistant and lying on a horizontal plane, $P_{S}$ equidistant from $P_{F}$ and $P_{B}, P_{D}$ oscillating around $O_{1}$, equidistant from $P_{F}$ and $P_{B}$ and coplanar to the latter points and $P_{B}$ (see Figure 1 ).

The system represents a simple model for the motion of a bicycle, as exhibited in [5]: the mass in $P_{S}$ is added on order to sketch the rigid structure of the bicycle (just as $P_{F}$ and $P_{B}$ represent the front and the back wheels), as well as the pendulum $P_{D}$ simulates the movement of a driver.

Let $O$ be a fixed point on the horizontal plane containing $P_{F}$ and $P_{B}, \mathbf{k}$ the ascending vertical versor, $C$ the midpoint of the segment $P_{B} P_{F}$ and $O_{1} C$ perpendicular to the same segment: the geometrical constraints (1) are written by means of the constant assigned values $\rho, \kappa, \kappa_{1}$ as

$$
\begin{aligned}
& \overline{P_{F} P_{B}}=2 \rho, \quad \overline{P_{S} C}=\kappa_{1}, \quad \overline{O_{1} P_{D}}=\kappa, \quad\left(P_{F}-O\right) \cdot \mathbf{k}=0, \quad\left(P_{B}-O\right) \cdot \mathbf{k}=0, \\
& \left(P_{S}-C\right) \cdot\left(P_{F}-P_{B}\right)=0, \quad\left(P_{D}-C\right) \cdot\left(P_{F}-P_{B}\right)=0
\end{aligned}
$$

Since the constraints are independent and $n=4$, we have $\mu=7, \ell=5$. Setting a fixed reference system $\{O,\langle\mathbf{i}, \mathbf{j}, \mathbf{k}\rangle\}$ and the angle $\phi$ between $P_{F}-P_{B}$ and $\mathbf{i}$, the angle $\theta$ between $P_{S}-C$ and $\mathbf{k}$, the angle $\theta_{1}$ between $P_{D}-O_{1}$ and $-\mathbf{k}$, one defines the orthonormal versors

$$
\mathbf{e}_{\phi}=\cos \phi \mathbf{i}+\sin \phi \mathbf{j}, \quad \mathbf{e}_{\theta}=-\sin \theta \sin \phi \mathbf{i}+\sin \theta \cos \phi \mathbf{j}+\cos \theta \mathbf{k}, \quad \mathbf{e}_{\theta_{1}}=-\sin \theta_{1} \sin \phi \mathbf{i}+\sin \theta_{1} \cos \phi \mathbf{j}-\cos \theta_{1} \mathbf{k}
$$

so that $P_{F}-P_{B}=2 \rho \mathbf{e}_{\phi}, P_{S}-C=\kappa_{1} \mathbf{e}_{\theta}, O_{1}-C=\left(\kappa_{1}+\kappa_{2}\right) \mathbf{e}_{\theta}, P_{2}-O_{1}=\kappa \mathbf{e}_{\theta_{1}}$ and choose the five parameters $\mathbf{q}=\left(x_{C}, y_{C}, \phi, \theta, \theta_{1}\right)$ as Lagrangian coordinates, where $x_{C} \mathbf{i}+y_{C} \mathbf{j}=C-O$.

Opting for considering the segment $P_{B} P_{F}$ as a rigid bar of mass $M$ (instead of a discrete point system, although not significant), the Lagrangian function (8) is written with $b=0, \quad c=0, U=-v_{1} g \cos \theta+\kappa m_{D} g \cos \theta_{1}$ and 


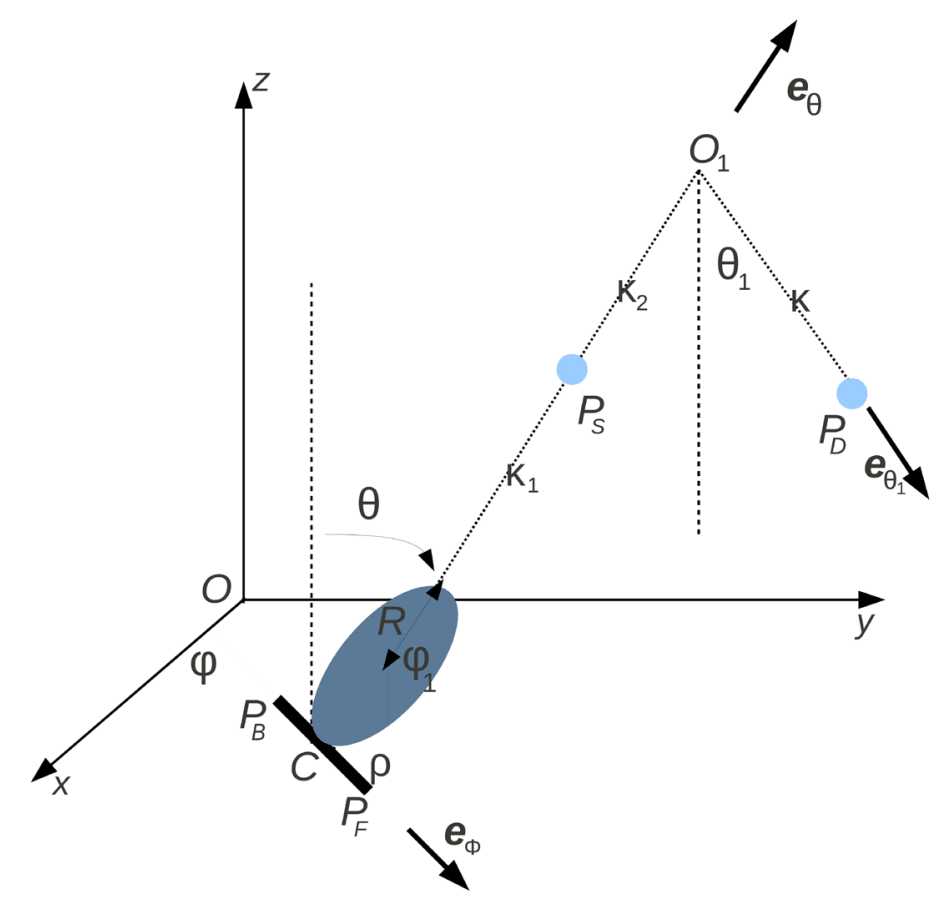

Figure 1. A simple model for the motion of a bicycle.

$$
A=\left(\begin{array}{ccccc}
M_{T} & 0 & -F_{1}\left(\theta, \theta_{1}\right) \cos \phi & -v_{1} \cos \theta \sin \phi & -m_{D} \kappa \cos \theta_{1} \sin \phi \\
0 & M_{T} & -F_{1}\left(\theta, \theta_{1}\right) \sin \phi & v_{1} \cos \theta \cos \phi & m_{D} \kappa \cos \theta_{1} \cos \phi \\
-F_{1}\left(\theta, \theta_{1}\right) \cos \phi & -F_{1}\left(\theta, \theta_{1}\right) \sin \phi & F_{2}\left(\theta, \theta_{1}\right) & 0 & 0 \\
-v_{1} \cos \theta \sin \phi & v_{1} \cos \theta \cos \phi & 0 & v_{2} & v_{3} \cos \left(\theta+\theta_{1}\right) \\
-m_{D} \kappa \cos \theta_{1} \sin \phi & m_{D} \kappa \cos \theta_{1} \cos \phi & 0 & v_{3} \cos \left(\theta+\theta_{1}\right) & m_{D} \kappa^{2}
\end{array}\right)
$$

where $M_{T}=M+m_{S}+m_{D}$ is the total mass and

$$
\left\{\begin{array}{l}
v_{1}=m_{S} \kappa_{1}+m_{D}\left(\kappa_{1}+\kappa_{2}\right), \quad v_{2}=m_{S} \kappa_{1}^{2}+m_{D}\left(\kappa_{1}+\kappa_{2}\right)^{2}, \quad v_{3}=m_{D} \kappa\left(\kappa_{1}+\kappa_{2}\right), \\
F_{1}\left(\theta, \theta_{1}\right)=v_{1} \sin \theta+m_{D} \kappa \sin \theta_{1}, \\
F_{2}\left(\theta, \theta_{1}\right)=I_{C}+m_{D} \kappa^{2} \sin ^{2} \theta_{1}+v_{2} \sin ^{2} \theta+2 m_{D} \kappa\left(\kappa_{1}+\kappa_{2}\right) \sin \theta \sin \theta_{1} .
\end{array}\right.
$$

The only one kinetic constraint concerns with the velocity of the back "wheel" $P_{B}$, to be aligned with the segment:

$$
\dot{P}_{B} \wedge \mathbf{e}_{\phi}=\mathbf{0}
$$

or $\dot{x}_{C} \sin \phi-\dot{y}_{C} \cos \phi+\frac{R}{2} \dot{\phi}=0$, that is (4) for $v=1, \alpha=\left(\sin \phi,-\cos \phi, \frac{R}{2}, 0,0\right), \quad \boldsymbol{\beta}=\mathbf{0}$.

Hence $\sigma=4$ and the four quasi-velocities (11) are selected by setting

$$
Z=\left(\begin{array}{ccccc}
\frac{1}{\rho} \cos \phi & \frac{1}{\rho} \sin \phi & 0 & 0 & 0 \\
-\frac{1}{\rho} \sin \phi & \frac{1}{\rho} \cos \phi & 0 & 0 & 0 \\
0 & 0 & 0 & 1 & 0 \\
0 & 0 & 0 & 0 & 1
\end{array}\right) \text { and } \boldsymbol{\psi}=\mathbf{0} .
$$


Furthermore, (12) gives

$$
\Gamma=\left(\begin{array}{cccc}
\rho \cos \phi & -\rho \sin \phi & 0 & 0 \\
\rho \sin \phi & \rho \cos \phi & 0 & 0 \\
0 & 1 & 0 & 0 \\
0 & 0 & 1 & 0 \\
0 & 0 & 0 & 1
\end{array}\right)
$$

so that

$$
A_{\Gamma}=\left(\begin{array}{cccc}
M_{T} \rho^{2} & -\rho F_{1} & 0 & 0 \\
-\rho F_{1} & F_{2}+\rho^{2} M_{T} & \rho v_{1} \cos \theta & \rho m_{D} \kappa \cos \theta_{1} \\
0 & \rho v_{1} \cos \theta & v_{2} & v_{3} \cos \left(\theta+\theta_{1}\right) \\
0 & \rho m_{D} \kappa \cos \theta_{1} & v_{3} \cos \left(\theta+\theta_{1}\right) & m_{D} \kappa^{2}
\end{array}\right)
$$

By computing the first line in (26) one finds the four equations of motion

$$
\begin{aligned}
& \rho^{2} M_{T} \dot{\eta}_{1}-\rho F_{1} \dot{\eta}_{2}-\left(F_{2}+\rho^{2} M_{T}\right) \eta_{2}^{2}+\rho F_{1} \eta_{1} \eta_{2}-\rho\left(v_{1} \cos \theta+\frac{\partial F_{1}}{\partial \theta}\right) \eta_{2} \eta_{3}-\rho\left(m_{D} \kappa \cos \theta_{1}+\frac{\partial F_{1}}{\partial \theta_{1}}\right) \eta_{2} \eta_{4}=0 \\
& -\rho F_{1} \dot{\eta}_{1}+\left(F_{2}+\rho^{2} M_{T}\right) \dot{\eta}_{2}+\rho v_{1} \dot{\eta}_{3} \cos \theta+\rho m_{D} \kappa \dot{\eta}_{4} \cos \theta_{1}+\rho F_{1} \eta_{1}^{2}-2 \rho F_{1} \eta_{2}^{2}-\rho v_{1} \eta_{3}^{2} \sin \theta-\rho m_{D} \kappa \eta_{4}^{2} \sin \theta_{1} \\
& \quad+\left(\rho^{2} M_{T}-F_{2}\right) \eta_{1} \eta_{2}-\rho \frac{\partial F_{1}}{\partial \theta}\left(\eta_{1} \eta_{3}+\eta_{1} \eta_{4}\right)+\frac{\partial F_{2}}{\partial \theta}\left(\eta_{2} \eta_{3}+\eta_{2} \eta_{4}\right)=0 \\
& \rho v_{1} \dot{\eta}_{2} \cos \theta+v_{2} \dot{\eta}_{3}+m_{D} \kappa\left(\kappa_{1}+\kappa_{2}\right) \dot{\eta}_{4} \cos \left(\theta+\theta_{1}\right)+\left(\rho v_{1} \cos \theta-\frac{1}{2} \frac{\partial F_{2}}{\partial \theta}\right) \eta_{2}^{2}-v_{3} \eta_{4}^{2} \sin \left(\theta+\theta_{1}\right)+\rho \frac{\partial F_{1}}{\partial \theta} \eta_{1} \eta_{2} \\
& \quad+\left(v_{2}-\frac{\rho}{2} v_{1} \sin \theta\right) \eta_{2} \eta_{3}+v_{3} \eta_{2} \eta_{4} \cos \left(\theta+\theta_{1}\right)-v_{1} g \sin \theta=0, \\
& m_{D} \kappa \rho \cos \theta_{1} \dot{\eta}_{2}+v_{3} \dot{\eta}_{3} \sin \left(\theta+\theta_{1}\right)+m_{D} \kappa^{2} \dot{\eta}_{4}+\left(\rho m_{D} \kappa \cos \theta-\frac{1}{2} \frac{\partial F_{2}}{\partial \theta_{1}}\right) \eta_{2}^{2}-v_{3} \eta_{3}^{2} \sin \left(\theta+\theta_{1}\right)+\rho \frac{\partial F_{1}}{\partial \theta_{1}} \eta_{1} \eta_{2} \\
& \quad+v_{3} \eta_{2} \eta_{3} \cos \left(\theta+\theta_{1}\right)+m_{D} \kappa^{2} \eta_{2} \eta_{4}+\kappa m_{D} g \sin \theta_{1}=0 .
\end{aligned}
$$

joined with the conservation of the quantity $\eta \cdot \nabla_{\eta} \tilde{\mathcal{L}}-\tilde{\mathcal{L}}$.

\subsection{Assignment of the Front Motion}

We modify the previous model by forcing the velocity of the front "wheel" to be a known function of time (a simpler version was considered in [6] for the motion of a bike): $P_{F}(t)-O=x_{F}(t) \mathbf{i}+y_{F}(t) \mathbf{j}$. With respect to (28), time $t$ enters explicitly the geometrical constraints and the fourth one has to be removed. Hence, in this example we have $n=3, \mu=6, \ell=3$ and we choose $\mathbf{q}=\left(\phi, \theta, \theta_{1}\right)$. The midpoint $C$ is located by $C-O=\left(x_{F}(t)-\rho \cos \phi\right) i+\left(y_{F}(t)-\rho \sin \phi\right) j$ and the Lagrangian function (8) is written with

$$
A=\left(\begin{array}{ccc}
F_{2}+\rho^{2} M_{T} & -\rho v_{1} \cos \theta & -\rho m_{D} \kappa \cos \theta_{1} \\
-\rho v_{1} \cos \theta_{1} & v_{2} & v_{3} \cos \left(\theta+\theta_{1}\right) \\
-\rho m_{D} \kappa \cos \theta_{1} & v_{3} \cos \left(\theta+\theta_{1}\right) & m_{D} \kappa^{2}
\end{array}\right), \quad \mathbf{b}=\left(\begin{array}{c}
\frac{1}{2} \rho M_{T} \boldsymbol{\beta}-F_{1} \frac{\partial \boldsymbol{\beta}}{\partial \phi} \\
-v_{1} \boldsymbol{\beta} \cos \theta \\
-m_{D} \kappa \boldsymbol{\beta} \cos \theta_{1}
\end{array}\right), \quad c=\frac{1}{2} M_{T}\left(\dot{x}_{F}^{2}(t)+\dot{y}_{F}^{2}(t)\right)
$$

whereas $U$ is the same function.

The constraint (30) is now $\dot{x}_{F}(t) \sin \phi-\dot{y}_{F}(t) \cos \phi+2 \rho \dot{\phi}=0$, that is (4) for $v=1, \alpha=(2 \rho, 0,0)$ 
$\boldsymbol{\beta}=\dot{x}_{F}(t) \sin \phi-\dot{y}_{F}(t) \cos \phi$. Choosing $\eta_{1} \dot{\theta}, \eta_{2}=\dot{\theta}_{1}$ we have simply

$$
Z=\left(\begin{array}{lll}
0 & 1 & 0 \\
0 & 0 & 1
\end{array}\right), \quad \Gamma=\left(\begin{array}{ll}
0 & 0 \\
1 & 0 \\
0 & 1
\end{array}\right), \quad \Theta=\left(\begin{array}{c}
1 /(2 \rho) \\
0 \\
0
\end{array}\right) \quad A_{\Gamma}=\left(\begin{array}{cc}
v_{2} & v_{3} \cos \left(\theta+\theta_{1}\right) \\
v_{3} \cos \left(\theta+\theta_{1}\right) & m_{D} \kappa^{2}
\end{array}\right)
$$

Equation (20) are written with

$$
Q=-v_{3} \sin \left(\theta+\theta_{1}\right)\left(\begin{array}{l}
\eta_{2}^{2} \\
\eta_{1}^{2}
\end{array}\right), \quad \Lambda=0, \quad N=-\frac{1}{4 \rho} \boldsymbol{\beta}\left(\frac{1}{2 \rho} \boldsymbol{\beta}\left(\begin{array}{c}
\frac{\partial F_{2}}{\partial \theta} \\
\frac{\partial F_{2}}{\partial \theta_{1}}
\end{array}\right)+\frac{\partial \boldsymbol{\beta}}{\partial \phi}\left(\begin{array}{c}
v_{1} \cos \theta \\
m_{D} \kappa \cos \theta_{1}
\end{array}\right)\right)
$$

and correspond to

$$
\begin{aligned}
v_{2} \dot{\eta}_{1}+v_{3} \dot{\eta}_{2} \cos \left(\theta+\theta_{1}\right)-v_{3} \sin \left(\theta+\theta_{1}\right) \eta_{2}^{2}-\frac{1}{8 \rho^{2}}\left(\dot{x}_{F}(t) \sin \phi-\dot{y}_{F}(t) \cos \phi\right)^{2} \frac{\partial F_{2}}{\partial \theta} \\
-\frac{1}{4 \rho}\left(\dot{x}_{F}(t) \sin \phi-\dot{y}_{F}(t) \cos \phi\right)\left(\dot{x}_{F}(t) \cos \phi+\dot{y}_{F}(t) \sin \phi\right) v_{1} \cos \theta-v_{1} g \sin \theta=0 \\
v_{3} \dot{\eta}_{1} \cos \left(\theta+\theta_{1}\right)+m_{D} \kappa^{2} \dot{\eta}_{2}-v_{3} \sin \left(\theta+\theta_{1}\right) \eta_{1}^{2}-\left(\dot{x}_{F}(t) \sin \phi-\dot{y}_{F}(t) \cos \phi\right)^{2} \frac{\partial F_{2}}{\partial \theta_{1}} \\
-\frac{1}{4 \rho}\left(\dot{x}_{F}(t) \sin \phi-\dot{y}_{F}(t) \cos \phi\right)\left(\dot{x}_{F}(t) \cos \phi+\dot{y}_{F}(t) \sin \phi\right) m_{D} \kappa \cos \theta_{1}+m_{D} \kappa g \sin \theta_{1}=0
\end{aligned}
$$

The energy balance (18) writes $\frac{\mathrm{d}}{\mathrm{d} t}\left(\eta \cdot \nabla_{\eta} \tilde{\mathcal{L}}-\tilde{\mathcal{L}}\right)=\Theta \beta \cdot \nabla_{\mathbf{q}} \tilde{\mathcal{L}}-\frac{\partial \tilde{\mathcal{L}}}{\partial t}$ and the function in the right side of the latter equality is

$$
\frac{1}{2} \Psi\left(\frac{1}{2} \boldsymbol{\beta}\left(M_{T}-\frac{F_{2}}{2 \rho^{2}}\right)-\frac{F_{1}}{\rho} \frac{\partial \boldsymbol{\beta}}{\partial \phi}-\eta_{1} v_{1} \cos \theta-\eta_{2} m_{D} \kappa \cos \theta_{1}\right)-\frac{1}{2 \rho} F_{1} \boldsymbol{\beta}\left(\frac{\partial \Psi}{\partial \phi}+\frac{1}{2 \rho}\left(\frac{\partial \boldsymbol{\beta}}{\partial \phi}\right)^{2}\right)+M_{T}\left(\dot{x}_{F} \ddot{x}_{F}+\dot{y}_{F} \ddot{y}_{F}\right)
$$

with $\Psi=\left(\frac{\partial \boldsymbol{\beta}}{\partial t}-\frac{\boldsymbol{\beta}}{2 \rho} \frac{\partial \boldsymbol{\beta}}{\partial \phi}\right)$.

\subsection{Rolling Disk with Pendulum}

A different version of the model 3.1 lies in replacing the bar with a disk and obtaining the unicycle with rider model presented in [7] (see Figure 1 again, replacing the bar with the disk). The system we consider here is a disk of diameter $2 R$ and mass $M$, in addition to the same points $P_{S}$ (with mass $m_{S}$ ) and $P_{D}$ (with mass $\left.P_{D}\right)$. We directly choose the coordinates (see Remark 2.1) $\mathbf{q}=\left(x_{C}, y_{C}, \phi, \phi_{1}, \theta, \theta_{1}\right)$ where the new parameter $\phi_{1}$ is the angle of rotation of the disk around the axis perpendicular to the disk and passing through the centre. The Lagrangian function is written with $U=-\hat{v}_{1} g \cos \theta+m_{D} g \kappa \cos \theta_{1}$ and

$$
A=\left(\begin{array}{cccccc}
M_{T} & 0 & -\hat{F}_{1} \cos \phi & 0 & -\hat{v}_{1} \cos \theta \sin \phi & -m_{D} \kappa \cos \theta_{1} \sin \phi \\
0 & M_{T} & -\hat{F}_{1} \sin \phi & 0 & \hat{v}_{1} \cos \theta \cos \phi & -m_{D} \kappa \cos \theta_{1} \cos \phi \\
-\hat{F}_{1} \cos \phi & -\hat{F}_{1} \sin \phi & \hat{F}_{2} & -I_{D} \sin \theta & 0 & 0 \\
0 & 0 & -I_{D} \sin \theta & I_{D} & 0 & 0 \\
-\hat{v}_{1} \cos \theta \sin \phi & \hat{v}_{1} \cos \theta \cos \phi & 0 & 0 & \hat{v}_{2}+\frac{1}{2} I_{D} & v_{3} \cos \left(\theta+\theta_{1}\right) \\
-m_{D} \kappa \cos \theta_{1} \sin \phi & -m_{D} \kappa \cos \theta_{1} \cos \phi & 0 & 0 & v_{3} \cos \left(\theta+\theta_{1}\right) & m_{D} \kappa^{2}
\end{array}\right)
$$


where $I_{D}=\frac{1}{2} M R^{2}$ and (see (29))

$$
\begin{array}{ll}
\hat{v}_{1}=v_{1}+M R, & \hat{v}_{2}=v_{2}+M R^{2}, \\
\hat{F}_{1}\left(\theta, \theta_{1}\right)=F_{1}\left(\theta, \theta_{1}\right)+M R \sin \theta, & \hat{F}_{2}\left(\theta, \theta_{1}\right)=F_{2}\left(\theta, \theta_{1}\right)-I_{C}+\left(M R^{2}+\frac{1}{2} I_{D}\right) \sin ^{2} \theta+\frac{1}{2} I_{D} .
\end{array}
$$

The kinematic constraint of rolling without sliding entails the zero velocity of the contact point $C$ :

$$
\dot{x}_{C}=\dot{\phi}_{1} R \cos \phi, \quad \dot{y}_{C}=\dot{\phi}_{1} R \sin \phi
$$

which is (4) with $v=2, \quad \alpha=\left(\begin{array}{llllll}1 & 0 & 0 & -R \cos \phi & 0 & 0 \\ 0 & 1 & 0 & -R \sin \phi & 0 & 0\end{array}\right)$ and $\boldsymbol{\beta}=\mathbf{0}$.

This time $\sigma=4$ and the choice

$$
\eta_{1}=\frac{\partial L}{\partial \phi}=\hat{F}_{2} \dot{\phi}-I_{D} \dot{\phi}_{1} \sin \theta-\hat{F}_{1}\left(\dot{x}_{C} \cos \phi+\dot{x} y_{C} \cos \phi\right), \quad \eta_{2}=\frac{\partial L}{\partial \phi}=I_{D} \dot{\phi}_{1}-I_{D} \dot{\phi} \sin \theta, \quad \eta_{3}=\dot{\theta}, \quad \eta_{4}=\dot{\theta}_{1}
$$

leads to

$$
\begin{aligned}
Z & =\left(\begin{array}{cccccc}
-\hat{F}_{1} \cos \phi & -\hat{F}_{1} \sin \phi & \hat{F}_{2} & -I_{D} \sin \theta & 0 & 0 \\
0 & 0 & -I_{D} \sin \theta & I_{D} & 0 & 0 \\
0 & 0 & 0 & 0 & 1 & 0 \\
0 & 0 & 0 & 0 & 0 & 1
\end{array}\right), \\
\Gamma & =\frac{1}{\delta}\left(\begin{array}{cccc}
I_{D} R \sin \theta \cos \phi & R \hat{F}_{2} \cos \phi & 0 & 0 \\
I_{D} R \sin \theta \sin \phi & R \hat{F}_{2} \sin \phi & 0 & 0 \\
I_{D} & I_{D} \sin \theta+R \hat{F}_{1} & 0 & 0 \\
I_{D} \sin \theta & \hat{F}_{2} & 0 & 0 \\
0 & 0 & \delta & 0 \\
0 & 0 & 0 & \delta
\end{array}\right)
\end{aligned}
$$

where $\delta\left(\theta, \theta_{1}\right)=I_{D} \hat{F}_{2}-I_{D} \sin \theta\left(I_{D} \sin \theta+R \hat{F}_{1}\right)>0$. Moreover

$$
A_{\Gamma}=\left(\begin{array}{cccc}
b_{1,1} & b_{1,2} & 0 & 0 \\
b_{2,1} & b_{2,2} & 0 & 0 \\
0 & 0 & \hat{v}_{2}+\frac{1}{2} I_{D} & v_{3} \cos \left(\theta+\theta_{1}\right) \\
0 & 0 & v_{3} \cos \left(\theta+\theta_{1}\right) & \hat{v}_{2}+\frac{1}{2} I_{D}
\end{array}\right)
$$

with

$$
\left\{\begin{array}{l}
b_{1,1}\left(\theta, \theta_{1}\right)=\frac{I_{D}}{\delta}\left(1+\frac{I_{D} \sin \theta}{\delta}\left(M_{T} R^{2} \sin \theta-R \hat{F}_{1}\right)\right) \\
b_{1,2}\left(\theta, \theta_{1}\right)=b_{2,1}\left(\theta, \theta_{1}\right)=\frac{I_{D} \sin \theta}{\delta}\left(1+\frac{R}{\delta}\left[M_{T} R \hat{F}_{2}-\left(I_{D} \sin \theta+R \hat{F}_{1}\right) \hat{F}_{1}\right]\right) \\
b_{2,2}\left(\theta, \theta_{1}\right)=\frac{\hat{F}_{2}}{\delta}\left(1+\frac{R}{\delta}\left[M_{T} R \hat{F}_{2}-\left(I_{D} \sin \theta+R \hat{F}_{1}\right) \hat{F}_{1}\right]\right)
\end{array}\right.
$$

and the corresponding equations of motion (20) are 


$$
\begin{aligned}
& b_{1,1} \dot{\eta}_{1}+b_{1,2} \dot{\eta}_{2}+\eta_{1} \eta_{3}\left(b_{1,1} G_{1}-b_{1,2} G_{5}+\frac{\partial b_{1,1}}{\partial \theta}\right)+\eta_{1} \eta_{4}\left(b_{1,1} G_{2}+\frac{\partial b_{1,1}}{\partial \theta_{1}}\right)+\eta_{2} \eta_{3}\left(b_{2,1} G_{1}-b_{2,2} G_{5}+\frac{\partial b_{2,1}}{\partial \theta}\right) \\
& +\eta_{2} \eta_{4}\left(b_{2,1} G_{2}+\frac{\partial b_{2,1}}{\partial \theta_{1}}\right)=0, \\
& b_{2,1} \dot{\eta}_{1}+b_{2,2} \dot{\eta}_{2}-\eta_{1} \eta_{3}\left(b_{1,1} G_{3}+b_{1,2} G_{6}-\frac{\partial b_{1,2}}{\partial \theta}\right)+\eta_{1} \eta_{4}\left(b_{1,1} G_{4}-\frac{\partial b_{1,2}}{\partial \theta_{1}}\right)-\eta_{2} \eta_{3}\left(b_{2,1} G_{4}+b_{2,2} G_{6}-\frac{\partial b_{2,2}}{\partial \theta}\right) \\
& \quad+\eta_{2} \eta_{4}\left(-b_{2,1} G_{4}+\frac{\partial b_{2,2}}{\partial \theta_{1}}\right)=0, \\
& \left(\hat{v}_{2}+\frac{1}{2} I_{D}\right) \dot{\eta}_{3}-v_{3} \dot{\eta}_{4} \cos \left(\theta+\theta_{1}\right)-\eta_{1}^{2}\left(b_{1,1} G_{1}+\frac{1}{2} \frac{\partial b_{1,1}}{\partial \theta}\right)+\eta_{2}^{2}\left(b_{2,1} G_{3}+b_{2,2} G_{6}-\frac{1}{2} \frac{\partial b_{2,2}}{\partial \theta}\right) \\
& \quad-\eta_{1} \eta_{2}\left(b_{2,1} G_{1}-b_{1,1} G_{3}-b_{2,2} G_{5}-b_{1,2} G_{6}+\frac{\partial b_{2,1}}{\partial \theta}\right)-v_{3} \eta_{4}^{2} \sin \left(\theta+\theta_{1}\right)-\hat{v}_{1} g \sin \theta=0, \\
& -v_{3} \dot{\eta}_{3} \cos \left(\theta+\theta_{1}\right)+m_{D} \kappa^{2} \dot{\eta}_{4}-\eta_{1}^{2}\left(b_{1,1} G_{2}+\frac{1}{2} \frac{\partial b_{1,1}}{\partial \theta_{1}}\right)+\eta_{2}^{2}\left(b_{2,1} G_{4}-\frac{1}{2} \frac{\partial b_{2,2}}{\partial \theta_{1}}\right) \\
& \quad+\eta_{1} \eta_{2}\left(b_{2,1} G_{2}-b_{1,1} G_{4}+\frac{\partial b_{2,1}}{\partial \theta_{1}}\right)-v_{3} \eta_{3}^{2} \sin \left(\theta+\theta_{1}\right)+m_{D} \kappa \sin \theta_{1}=0 .
\end{aligned}
$$

where

$$
\begin{aligned}
& G_{1}\left(\theta, \theta_{1}\right)=\frac{I_{D}}{\delta}\left(\frac{\partial \hat{F}_{2}}{\partial \theta}-\left(I_{D}+R \hat{v}_{1}\right) \cos \theta \sin \theta\right), \\
& G_{2}\left(\theta, \theta_{1}\right)=\frac{I_{D}}{\delta}\left(\frac{\partial \hat{F}_{2}}{\partial \theta_{1}}-m_{D} \kappa R \cos \theta_{1} \sin \theta\right), \\
& G_{3}\left(\theta, \theta_{1}\right)=\frac{1}{\delta}\left(\left(I_{D}+R \hat{v}_{1}\right) \hat{F}_{2} \cos \theta-\left(I_{D} \sin \theta+R \hat{F}_{1}\right) \frac{\partial \hat{F}_{2}}{\partial \theta}\right), \\
& G_{4}\left(\theta, \theta_{1}\right)=\frac{1}{\delta}\left(m_{D} \kappa R \hat{F}_{2} \cos \theta_{1}-\left(I_{D} \sin \theta+R \hat{F}_{1}\right) \frac{\partial \hat{F}_{2}}{\partial \theta_{1}}\right), \\
& G_{5}\left(\theta, \theta_{1}\right)=\frac{I_{D}^{2}}{\delta} \cos \theta, \\
& G_{6}\left(\theta, \theta_{1}\right)=\frac{I_{D}}{\delta}\left(I_{D} \sin \theta+R \hat{F}_{1}\right) \cos \theta .
\end{aligned}
$$

\subsection{Assigned Rotational Velocity of the Disk}

We finally consider the same system with the differential constraint (31), but $\phi_{1}=\phi_{1}(t)$ assigned (we may think about an engine-driven motor bike or electric bike): in that case $\mathbf{q}=\left(x_{C}, y_{C}, \phi, \theta, \theta_{1}\right)$ and (4) is setted with $v=2$ and $\alpha=\left(\begin{array}{ccccc}1 & 0 & 0 & 0 & 0 \\ 0 & 1 & 0 & 0 & 0\end{array}\right), \quad \beta=\left(\begin{array}{c}-\dot{\phi}_{1}(t) R \cos \phi \\ -\dot{\phi}_{1} R \sin \phi\end{array}\right)$.

The Lagrangian fucntion (8) is written with $A$ the same as in the previous Example 3.1, except for removing the fourth row and the fourth column, and $\mathbf{b}=\left(\begin{array}{c}0 \\ 0 \\ -I_{D} \sin \theta \\ 0 \\ 0\end{array}\right), \quad c=\frac{1}{2} I_{D} \dot{\phi}_{1}^{2}((t))$. In the matter of (11), which has to 
be written for $\sigma=3$, if one defines the quasi-velocities $\eta_{1}=\frac{\partial L}{\partial \phi}=\hat{F}_{2} \dot{\phi}-\hat{F}_{1}\left(\dot{x}_{C} \cos \phi+\dot{x} y_{C} \cos \phi\right)-I_{D} \dot{\phi}_{1}(t) \sin \theta$, $\eta_{2}=\dot{\theta}, \quad \eta_{3}=\dot{\theta}_{1}$ one gets $\psi=\mathbf{0}$ and

$$
Z=\left(\begin{array}{ccccc}
-\hat{F}_{1} \cos \phi & -\hat{F}_{1} \sin \phi & \hat{F}_{2} & 0 & 0 \\
0 & 0 & 0 & 1 & 0 \\
0 & 0 & 0 & 0 & 1 \\
1 & 0 & 0 & 0 & 0 \\
0 & 1 & 0 & 0 & 0
\end{array}\right), \quad \Gamma=\left(\begin{array}{ccc}
0 & 0 & 0 \\
0 & 0 & 0 \\
\frac{1}{\hat{F}_{2}} & 0 & 0 \\
0 & 1 & 0 \\
0 & 0 & 1
\end{array}\right), \quad \Theta=\left(\begin{array}{cc}
1 & 0 \\
0 & 1 \\
\frac{\hat{F}_{1}}{\hat{F}_{2}} \cos \phi & \frac{\hat{F}_{1}}{\hat{F}_{2}} \sin \phi \\
0 & 0 \\
0 & 0
\end{array}\right)
$$

Calculating the products in (15) gives

$$
\begin{aligned}
A_{\Gamma} & =\left(\begin{array}{ccc}
\frac{1}{\hat{F}_{2}} & 0 & 0 \\
0 & \hat{v}_{2}+\frac{1}{2} I_{D} & v_{3} \cos \left(\theta+\theta_{1}\right) \\
0 & v_{3} \cos \left(\theta+\theta_{1}\right) & m_{D} \kappa^{2}
\end{array}\right), \\
A_{\Gamma, \Theta} & =\left(\begin{array}{cc}
0 & 0 \\
-\hat{v}_{1} \cos \theta \sin \phi & \hat{v}_{1} \cos \theta \cos \phi \\
-m_{D} \kappa \cos \theta_{1} \sin \phi & m_{D} \kappa \cos \theta_{1} \cos \phi
\end{array}\right), \\
A_{\Theta} & =\left(\begin{array}{cc}
M_{T}-\frac{\hat{F}_{1}^{2}}{\hat{F}_{2}} \cos { }^{2} \phi & -\frac{\hat{F}_{1}^{2}}{\hat{F}_{2}} \sin \phi \cos \phi \\
-\frac{\hat{F}_{1}^{2}}{\hat{F}_{2}} \sin \phi \cos \phi & M_{T}-\frac{\hat{F}_{1}^{2}}{\hat{F}_{2}} \sin ^{2} \phi
\end{array}\right)
\end{aligned}
$$

and the computation of (20) gives the three equations of motion

$$
\begin{aligned}
& \frac{1}{\hat{F}_{2}} \dot{\eta}_{1}+\frac{1}{\hat{F}_{2}} I_{D} \dot{\phi}_{1}(t) \eta_{2} \cos \theta+\dot{\phi}_{1}(t) R \frac{1}{\hat{F}_{2}}\left(\hat{v}_{1} \eta_{2} \cos \theta+m_{D} \kappa \eta_{3} \cos \theta_{1}\right)-\frac{I_{D}}{\hat{F}_{2}} \dot{\phi}_{1}(t) \eta_{2} \cos \theta+\frac{\hat{F}_{1}}{\hat{F}_{2}^{2}} I_{D} \dot{\phi}_{1}^{2}(t) R \cos \theta=0, \\
& \frac{1}{\hat{F}_{2}^{2}}\left(\left(\hat{v}_{2}+\frac{1}{2} I_{D}\right) \dot{\eta}_{2}+v_{3} \cos \left(\theta+\theta_{1}\right) \dot{\eta}_{3}\right)-I_{D} \frac{1}{\hat{F}_{2}^{2}} \frac{\partial \hat{F}_{2}}{\partial \theta} \dot{\phi}_{1}(t) \eta_{1} \sin \theta+R \dot{\phi}_{1}(t) \frac{1}{\hat{F}_{2}} \eta_{1} \hat{v}_{1} \cos \theta-R \dot{\phi}_{1}(t) \frac{\hat{F}_{1}}{\hat{F}_{2}^{2}} \eta_{1} \frac{\partial \hat{F}_{2}}{\partial \theta} \\
& \quad+\frac{I_{D}}{\hat{F}_{2}} \dot{\phi}_{1}(t) \eta_{1} \cos \theta+\dot{\phi}_{1}^{2}(t)\left(\frac{1}{2} R^{2} \frac{\partial}{\partial \theta}\left(\frac{\hat{F}_{1}^{2}}{\hat{F}_{2}}\right)+\frac{I_{D}}{\hat{F}_{2}}\left(\left(2 I_{D}+R \hat{v}_{1}\right) \sin \theta \cos \theta-2 \frac{\hat{F}_{1}}{\hat{F}_{2}} \frac{\partial \hat{F}_{2}}{\partial \theta} R \sin \theta-\hat{F}_{1} R \cos \theta\right)\right) \\
& \quad-\hat{v}_{1} g \sin \theta=0, \\
& \frac{1}{\hat{F}_{2}^{2}}\left(v_{3} \cos \left(\theta+\theta_{1}\right) \dot{\eta}_{2}+m_{D} \kappa^{2} \dot{\eta}_{3}\right)+\frac{1}{\hat{F}_{2}} \dot{\phi}_{1}(t)\left(m_{D} \kappa R \cos \theta_{1}-\left(I_{D} \sin \theta+R \hat{F}_{1}\right) \frac{1}{\hat{F}_{2}} \frac{\partial \hat{F}_{2}}{\partial \theta_{1}}\right) \eta_{1} \\
& \quad+\dot{\phi}_{1}^{2}(t)\left(\frac{1}{2} R^{2} \frac{\partial}{\partial \theta_{1}}\left(\frac{\hat{F}_{1}^{2}}{\hat{F}_{2}}\right)+\frac{I_{D}}{\hat{F}_{2}} \sin \theta\left(m_{D} \kappa \cos \theta_{1}-2 R \frac{\hat{F}_{1}}{\hat{F}_{2}} \frac{\partial \hat{F}_{2}}{\partial \theta_{1}}\right)\right)+m_{D} \kappa g \sin \theta_{1}=0 .
\end{aligned}
$$

\section{Conclusions}

The paper aims at formulating a general scheme of equations for rheonomic mechanical systems exposed to ei- 
ther geometrical (1) and differential (2) constraints. We pay special attention to tell apart the different contributions due to the explicit dependence on time, deriving from the holonomous constrictions (via $\mathbf{b}$ and $c$ of (8)), the nonholonomous constrictions (via $\beta$ of (4)) and the definition of quasi-velocities (via $\psi$ ) of (11)).

Since the equations of motion are projected in the subspace of the velocities allowed by the constraints (both holonomous and nonholonomous), the Lagrange multipliers are absent from the equations.

The procedure proposed by (20) requires only calculation of the Jacobian matrix of vectors and the algebraic multiplication of matrices and vectors.

Making use of quasi-velocities renders the equations versatile to more than one formalism and, as it is known, the appropriate choice of them meets the target of facilitating the mathematical resolution of the problem.

The last point is part of the matters listed below and which will be dealt with in the future:

-Find an appropriate choice of the quasi-velocities in order to disentangle (20) from (13) as much as possible,

-Make use of the structure of the equations and of the properties of the various matrices involved in order to study the stability of the system,

-Take advantage of some peculiarity of the system in order to refine the set of equations and achieve information.

The latter subject is faced in [8] [9] for the stationary case by means of a robust and complex theory in connection with symmetries in nonholonomic systems.

\section{References}

[1] Poincaré, H. (1901) Sur une forme nouvelle des èquations de la méchanique. Comptes Rendus de l'Académie des Sciences, 132, 369-371.

[2] Gantmacher, F.R. (1975) Lectures in Analytical Mechanics. MIR.

[3] Maruskin, J.M. and Bloch, A.M. (2011) The Boltzman-Hamel Equations for the Optimal Control of Mechanical Systems with Nonholonomic Constraints. International Journal of Robust and Nonlinear Control, 21, 373-386. http://dx.doi.org/10.1002/rnc.1598

[4] Cameron, J.M. and Book, W.J. (1997) Modeling Mechanisms with Nonholonomic Joints Using the Boltzmann-Hamel Equations. Journal International Journal of Robotics Research, 16, 47-59. http://dx.doi.org/10.1177/027836499701600104

[5] Talamucci, F. (2014) The Lagrangian Method for a Basic Bicycle. Journal of Applied Mathematics and Physics, 2, 4660.

[6] Levi, M. (2014) Bike Tracks, Quasi-Magnetic Forces, and the Schrödinger Equation. SIAM News, 47.

[7] Zenkov, V., Bloch, A.M. and Mardsen, J.E. (2002) Stabilization of the Unicycle with Rider. Systems and Control Letters, 46, 293-302. http://dx.doi.org/10.1016/S0167-6911(01)00187-6

[8] Bloch, A.M., Krishnaprasad, P.S., Mardsen, J.E. and Murray, R. (1996) Nonholonomic Mechanical Systems with Symmetry. Archive for Rational Mechanics and Analysis, 136, 21-99. http://dx.doi.org/10.1007/BF02199365

[9] Bloch, A.M., Mardsen, J.E. and Zenkov, D.V. (2009) Quasivelocities and Symmetries in Non-Holonomic Systems. Dynamical Systems, 24, 187-222. http://dx.doi.org/10.1080/14689360802609344 